\title{
Pseudo-Aneurisma Femoral Micótico após Onze Anos de Cateterismo
}

\author{
Mycotic Femoral Pseudoaneurysm after 11 years of Catheterism
}

Marcello Barbosa Barros, Arturo Almazán, Francisco S. Lozano

Unidade de Cirurgia Vascular do Hospital Universitário de Salamanca - Universidade de Salamanca - Salamanca, Espanha

Relatamos o caso incomum de um alpinista de 52 anos que apresentava dor e edema em sua coxa direita, o que revelou ser um pseudoaneurisma micótico roto, sem histórico de trauma recente ou outra causa aparente. O paciente relatou uma história de infarto do miocárdio onze anos antes, com a realização de dois cateterismos femorais para cineangiocoronariografia. Ele negou qualquer episódio de febre ou diagnóstico de bacteremia naquele momento ou mais tarde, como também outra queixa durante esses onze anos. A raridade do caso, a aparência dessa complicação extremamente tardia, juntamente com o tipo de atividade esportiva do paciente sugeriram-nos publicar o caso.
The authors report the unusual case of a 52-year-old mountain climber that presented with pain and swelling in his right thigh which revealed to be a ruptured mycotic pseudoaneurysm with no history of recent trauma or other apparent cause. The patient reported a past history of myocardial infarction 11 years before, with the performing of two femoral catheterisms for coronary angiography. He denied any episode of fever or diagnosis of bacteraemia at that time or later, nor any other complaint during these 11 years. The rarity of the case, the appearance of this extremely late complication together with the patient's kind of sportive activity prompted us to publish the case.
Cineangiocoronariografia é um procedimento complexo, invasivo e potencialmente prejudicial, indicado, na maioria das vezes, em situações como infarto do miocárdio. Podemos listar muitas complicações relacionadas a esse procedimento, como situações como a bacteremia, tromboembolismo, fístulas arteriovenosas, esvaziamento arterial, infecção arterial e formações de pseudo-aneurisma.

Pseudo-aneurismas femorais micóticos tipicamente aparecem em dois meses ou mais após violação arterial e geralmente em pacientes com história anterior de bacteremia e/ou cateterismo seguido de endocardite ${ }^{1}$.

Diagnóstico e tratamento precoce de pseudoaneurisma infectado são essenciais para prevenir ruptura ou embolização distal. Isso fez que avaliássemos um caso de apresentação atípica e extremamente tardia de um pseudo-aneurisma infectado em um alpinista de 52 anos de idade, onze anos depois da cateterização.

O paciente deu seu consentimento para a publicação.

\section{RELATO DO CASO}

Paciente branco, do sexo masculino, 52 anos de idade, com história prévia de infarto do miocárdio onze anos antes e hipertensão arterial, sob uso de aspirina e medicamentos antidepressivos, chegou ao pronto-socorro queixando-se de dor e inchaço na coxa direita, os quais haviam iniciado na semana anterior, enquanto escalava montanha. $\mathrm{O}$ paciente disse que teve febre de $39^{\circ} \mathrm{C}$ e calafrios no primeiro dia. O exame físico revelou um homem de aparência saudável, sem febre, com sua coxa direita com aumento do diâmetro, quente e sensível ao toque. A panturrilha encontrava-se normal. Pulsações estavam presentes em todos os níveis do membro inferior direito. 0 ultra-som abdominal não constatou anormalidades e no Doppler da artéria femoral direita esta foi relatada como normal naquele momento. Dados anormais não foram observados no perfil hematológico ou exames bioquímicos de sangue. O paciente foi então encaminhado para casa com analgésicos e antiinflamatórios.

Após uma semana, ele retornou ao hospital reclamando de dor aguda e mais inflamação na sua coxa direita. A dor irradiava desde a virilha até o joelho ao longo do lado medial da coxa correspondente. Manifestava anorexia e ter perdido $12 \mathrm{~kg}$ em duas semanas. Não houve surtos de febre ou calafrios. Durante o exame, o paciente apresentava-se em regular estado geral, pálido e sudoréico. Não foi registrada febre. A coxa direita estava edematosa, dura e bastante sensível ao toque. Uma massa pulsátil e dolorida era palpável na virilha direita, que se estendia até o terço superior da coxa, com seu limite superior não sendo sentido como se estendesse para cima, através do ligamento inguinal. 0 joelho estava flexionado 
a $45^{\circ}$ e muito dolorido para movimentos. Exames de sangue mostraram uma leve anemia, leucocitose com alta contagem neutrofílica. Exames bioquímicos não apresentaram anormalidades. 0 perfil de coagulação estava normal, bem como as radiografias abdominal e peitoral. O eletrocardiograma (ECG) mostrou sinais de antigo infarto de miocárdio de parede posterior.

Sob questionamento, referiu ter tido um infarto do miocárdio onze anos antes, com dois cateterismos femorais direitos com objetivo diagnóstico, sem complementação terapêutica. O paciente negou intercorrência na ocasião.

Nenhum sopro cardíaco anormal foi auscultado. 0 Doppler naquele momento mostrou um aneurisma femoral direito comum roto, bem como trombose das veias femorais direitas superficiais e comuns. Foi indicada intervenção cirúrgica imediata. Um pseudo-aneurisma femoral comum roto foi extraído e reparado com veia safena invertida a partir da perna contra-lateral modo término-terminal. Sinais de infecção local não eram aparentes. O ferimento foi fechado após irrigação com solução salina e povidona-iodo. Um dreno de sucção foi deixado no local.

No período pós-operatório, o paciente seguiu tratamento antibiótico com amoxicilina/ácido clavulínico. O coágulo do aneurisma foi reportado como colonizado por um Staphylococcus aureus sensível a meticilina. Febre e calafrios desenvolveram-se 72 horas no pós-operatório, com cultura de sangue apresentando, em três instâncias, S. aureus sensível a vancomicina. Um abscesso foi drenado do ferimento, com a cultura também apresentando o mesmo agente. Naquele momento, o antibiótico foi trocado por vancomicina e mantido por duas semanas. 0 ecocardiograma não apresentou sinais de endocardite ou vegetações. Quinze dias após a operação, a febre desapareceu e não houve repetição de calafrios. 0 ferimento cicatrizou bem em 25 dias e 0 paciente recebeu alta com um índice de Doppler tornozelobraquial de 1,0. Ele foi mantido com amoxicilina/ácido clavulânico via oral por um mês.

\section{Discussão}

Bacteremia após o cateterismo arterial percutâneo é uma complicação bastante conhecida, com o $S$. aureus sendo o responsável em 54\%-57\% dos casos ${ }^{1,2}$. Aneurismas micóticos da artéria femoral também são freqüentes, seguindo invasão percutânea e bacteremia, em razão de colonização de uma área íntima danificada, particularmente se for repetido ou se a presença do cateter perdurar por mais de dois dias. Outras etiologias são muito raras $^{3,4}$. Essa complicação parece ser mais freqüente em idosos, pacientes com doença arterial periférica ou imunodeficientes, aparecendo em alguns dias ou meses após o cateterismo. Geralmente, os pseudo-aneurismas se desenvolvem com rapidez, sendo potencialmente vulneráveis para ruptura. Da mesma maneira, diagnóstico precoce e tratamento são fundamentais. Esses aneurismas são, em geral, facilmente diagnosticados em bases clínicas. No entanto, em raras instâncias, a inflamação peri-aneurismática e nódulos linfáticos locais podem disfarçar a massa pulsátil, que pode ser erroneamente confundida com um abscesso ou trombose venosa ${ }^{5}$; um alto grau de suspeita é, então, essencial.

0 tratamento é urgente. As melhores opções são ressecção do aneurisma, com a eliminação de secreção purulenta, detritos e tecido morto, e reconstituição da circulação sangüínea com revascularizção autógena arterial ou venosa, seja in situ ou em um modo extra-anatômico. O reparo arterial in situ acarreta o risco de infecção de enxerto, até mesmo se for utilizado material protético e isso pode resultar em rompimento de anastomose ou trombose ${ }^{6}$. No caso de aneurismas femorais comuns ou aneurismas de artéria ilíaca comuns, o cirurgião poderá optar pela não-reconstrução no momento da primeira operação e realizá-la mais tarde, se necessário ${ }^{7}$. Antibióticos devem ser mantidos por, pelo menos, um mês. Esse ponto, no entanto, está aberto para discussão.

Nosso caso é incomum, por causa de sua apresentação tardia (onze anos após cateterismo arterial), sem história pregressa de bacteremia ou endocardite, ocorrido em jovem saudável atleta (alpinista). Um outro aspecto incomum foram os primeiros sinais clínicos confusos, sem massa categoricamente detectada que, em mãos inexperientes, atrasaram o diagnóstico.

Há controvérsia sobre o início da formação do pseudoaneurisma: no momento do cateterismo repetido, com uma possível bacteremia "silenciosa", ou a partir de contaminação local. A última possibilidade é menos provável pelo número de anos que se passaram. De maneira alternativa, considera-se que o cateterismo arterial rompeu minimamente a parede arterial, talvez até produzindo um pseudo-aneurisma assintomático, sendo mais tarde infectado por uma bacteremia assintomática de $S$. aureus. Essa possibilidade é freqüente, uma vez que o $S$. aureus é uma colonização cutânea comum ao homem. Von Eiff e cols. ${ }^{8}$ mostraram, com um intervalo de confiança de $95 \%$, que pelo menos $50 \%$ de bacteremia por S. aureus ocorreram em pacientes colonizados nas narinas anteriores com o mesmo clone que foi isolado no sangue. O S. aureus resistente a meticilina também foi cultivado a partir do nariz e axilas em $11 \%$ de um grupo de pacientes estudado por Lecomte e cols., bem como em $40 \%$ das lesões, tais como úlceras venosas ou pé diabético ${ }^{9}$. Outros pacientes sob risco de contaminação nasal com o S. aureus são aqueles com eczemas e psoríase ${ }^{10}$.

Tudo o que foi mencionado mostra-nos que a possibilidade de bacteremia por $S$. aureus a partir da contaminação nasal pode ocorrer freqüentemente, não apenas após cateterismo, mas também a partir de feridas de pele ou trato respiratório inferior ${ }^{8}$ e, esse pode ter sido 
o caso de nosso paciente. Nesse caso, profilaxia antibiótica poderia, talvez, ser de importância em pacientes de alto risco, tais como aqueles com úlceras venosas ou lesões de pé diabético, para eliminar fontes potenciais de $S$. aureus a partir do nariz, além de seguir as diretrizes básicas de profilaxia em radiologia intervencional.

\section{REFERÊNCIAS}

1. Ehni WF, Reller LB. Short-course therapy for catheter-associated Staphylococcus aureus bacteremia. Arch Intern Med 1989;149:533-36

2. Malanoski GJ, Samore MH, Pefanis A, Karchmer AW. Staphylococcus aureus catheter-associated bacteremia. Minimal effective therapy and unusual infectious complications associated with arterial sheath catheters. Arch Intern Med 1995;155:1161-66.

3. Woodrum DT, Welke KF, Fillinger MF. Candida infection associated with a solitary mycotic common iliac artery aneurysm. J Vasc Surg $1001 ; 34: 166-68$.

4. Bagilet D, Notario R, Busnelli L, Bertoletti F. Aneurisma femoral infectado por Campylobacter fetus. Med Clin 2001;116:237.

5. Fung Y, Ahn J, Stancato-Pasik A, et al. Atypical angiographic appearance of a superficial femoral artery mycotic aneurysm. Br J Radiol 1997;70:961-63.
6. Reddy DJ, Ernst CB. Infected aneurysms. In Rutherford RB (ed.) Vascular Surgery. $5^{\text {th }}$ ed. Philadelphia: W.B. Saunders, 2000, 1383-97.

7. Arora S, Weber MA, Fox CJ, Neville R, Lidor A, Sidawy AN. Common femoral artery ligation and local debridement: a safe treatment for infected femoral artery pseudoaneurysms. J Vasc Surg 2001;33:990-93.

8. Von Eiff C, Becker K, Machka K, Stammer H, Peters G. Nasal carriage as a source of Staphylococcus aureus bacteremia. Study Group. N Engl J Med 2001;344:11-16.

9. Lecomte F, Nouvellon M, Levesque H. Nasal carriage of Staphylococcus aureus. N Engl J Med 2001;344:1399-400.

10. Casewell MW. The nose: an underestimated source of Staphylococcus aureus causing wound infection. J Hosp Infect 1998; 40(Suppl B): S3-11. 